ISSN 1112-9867

Available online at

http://www.jfas.info

\title{
DETERMINING THE PARAMETERS OF CHIRP SIGNALS USING CYCLOSTATIONARY METHOD IN PRESENCE OF THE INTERFERENCE
}

\author{
K. Heydari ${ }^{1, *}$, P. Azmi ${ }^{2}$, B. Abbasi ${ }^{2}$ and A. Heydari ${ }^{3}$ \\ ${ }^{1}$ Department of Electrical Engineering, Tarbiat Modares University, Tehran \\ ${ }^{2}$ Faculty of Electrical Engineering, Tarbiat Modares University \\ ${ }^{3}$ Department of Electrical Engineering Shahid Beheshti University, Tehran
}

Published online: 09 June 2016

\begin{abstract}
This paper deals with the problems of detecting a chirp signal of motion target against a strong undesired signal from the transmitter in radar. We use Generalized AlmostCyclostationary (GACS) signal processing method to determine the rate and initial frequency of the chirp signal in presence of the strong undesired signal. This technique which exploits the second order cyclostationary to detect features of the chirp signal in low SNR has desirable properties. The study utilized a method to determine and characterize signals for highly adverse signal to interference plus noise and explain how using cyclostationary properties can extract features of chirp signal from the mixture received signal. The theory and simulation of this method indicated that the chirp rate depends on the lag parameter and the cycle frequency.
\end{abstract}

Keywords: Chirp Signal, Cyclostationary, initial frequency, Chirp rate.

Author Correspondence, e-mail: k.haydari@gmail.com

doi: http://dx.doi.org/10.4314/jfas.8vi2s.63

\section{INTRODUCTION}

The performance of Continuous Wave (CW) radar and pulsed radar is limited in the presence of clutter by the phase and amplitude noise. Local Oscillator (LO) used for frequency conversion to Intermediate Frequency (IF) has an important effect on the phase noise. The 
phase noise is the most important degradation effect radar and it influences the performance of the radar in several ways. The main issue is that the radar transmits and receives simultaneously. Thus, the signal will leak directly from the transmitter to the receiver. This will happen through space and even more severely through the substrate if the transmitter and the receiver share the same circuit board. The undesired signal may be so strong that it may saturate either the Low-Noise Amplifier (LNA) or mixer. However, a more common problem is that the phase noise that leaks as well will have a tendency to mask out targets as the targets are detected at frequency offsets of the carrier. The same is the case for the strong nearby clutter.

The performance of these radars can be improved by elimination or reduction of noise. Different noise cancellation methods are presented in radar system, in 0 the author has developed a method of characterizing the effect range correlation on phase noise spectrum. As a stochastic process formulation was employed, the method was unique and made for a characterization that clearly illustrated various factors that constitute the spectrum at signal processor input. Based on 0 master oscillator requirements for coherent radar application are derived from consideration of mainlobe width and sidelobe. These techniques measured oscillator stability characteristics that are useful for radar system by taking periodic and random phase modulation into account.

In the method used in [1] the effects on the receiver sensitivity of transmitter phase noise reflected from very large radar (pulse radar) targets are taken into account. This paper looked briefly at representing the detection of small targets against a high density clutter background and considered the phase noise cancellation equation. At last, it is shown that the phase noise can be minimized by matching path length around the reception mixer. Detection methods based on Maximum Likelihood (ML) and Maximum M Posterior (MAP) are very convenient methods although they have complicated computations in low SNR [2]. In this paper, chirp rate and initial frequency are determined in presence of the undesired signal using feature of generalized almost-cyclostationary method. Detection of low SNR signal in presence of the perturbation signals can be improved by extracted features.

The rest of the paper is organized as follows: section II presents the system model; section III formula and analyses the method; section IV presents the simulation results; and section V summarizes the conclusions. 


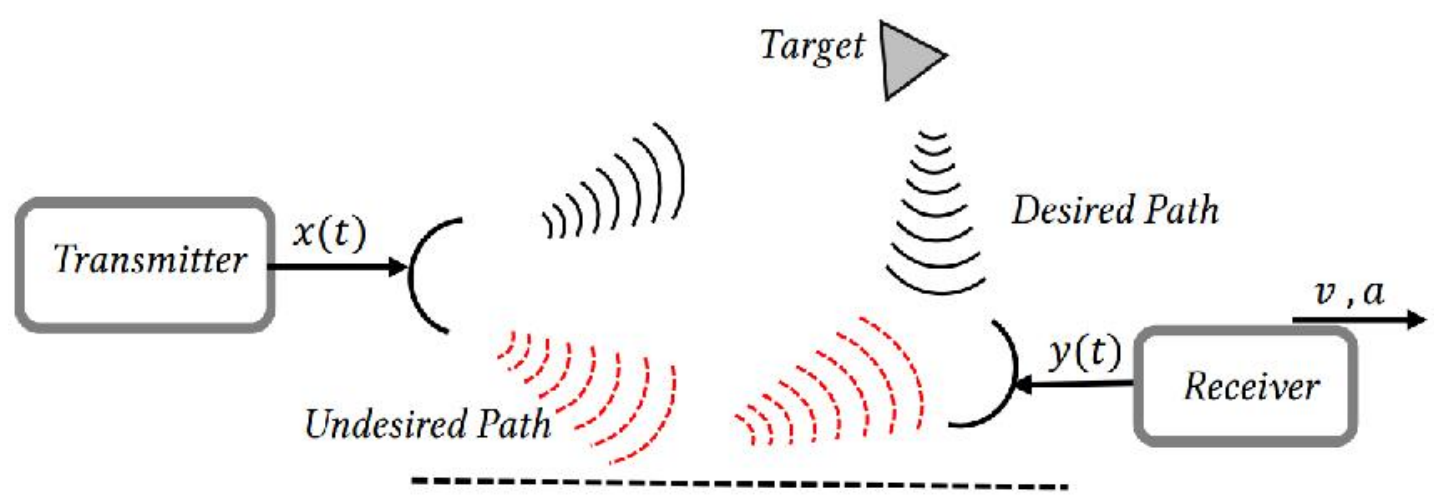

Fig.1. System model with undesired path and desired path

\section{SYSTEM MODEL}

A block diagram of radar configuration considered in this paper is shown in Fig.1. The amplitude and phase noise are what give rise to amplitude and phase noise sideband on transmitted signal, and cause spectral spreading of the transmitted signal that was discussed in introduction. The transmitter elements modulator, amplifiers, power tube, etc. also add noise to transmitted signal but their contributions are usually negligible in comparison with the LO. A normalized signal that is transmitted can be represented by

$x(t)=\operatorname{Re}\left\{\tilde{x}(t) \exp \left(-j 2 \pi f_{c}\right)\right\}$

Where $\operatorname{Re}\{$.$\} denotes real part, f_{c}$ is the carrier frequency, and $\tilde{x}(t)$ is a complex signal that has amplitude noise. As mentioned, the phase noise causes the spectral spreading of the transmitted signal. For simplicity, the phase noise is considered in $\tilde{x}(t)$.

\section{FORMULA AND ANALYSES}

In the model presented in this paper for the case of relative motion between target and receiver when the relative radial speed $v$ can be constant within the observation interval $\left(t_{0}, t_{0}+T\right)$ and by addressing [3] the following condition should be provided

$$
B T \ll\left|1+\frac{f}{v}\right|
$$

Where $B$ is bandwidth of $x(t)$ and $T$ is the length of observation interval. If condition (2) is satisfied, it is generally stated that the signal is narrow-band condition. Considering the system model in Fig.1, the received signal in radar can be represented by

$$
y_{r}(t)=y_{d}(t)+y_{u}(t)
$$

Where $y_{u}$ and $y_{d}$ receive signal from the undesired path and desired path, respectively. 


\section{GENERALIZED ALMOST-CYCLOSTATIONARY}

Many processes in nature arise from the interaction of periodic phenomena with random phenomena. The results are processes which lack being periodic but their statistical functions are periodic functions of time. These processes are called cyclostationary and are an appropriate mathematical model for signals encountering in radar, sonar, etc. [4]. In communications, radar and sonar, the cyclostationary properties of signals have been exploited to counteract the effect of noise and interference in weak signal detection problems[5].

Continuous signal $x(t)$ with zero mean is called second order cyclostationary if its time varying autocorrelation function $R_{x x^{*}}(t, \tau)$ is defined as

$$
R_{x x^{*}}(t, \tau)=E\left\{x^{*}(t) x(t+\tau)\right\}
$$

For each lag parameter $\tau$ is periodic in time $t$ and it can be represented as Fourier series

$$
R_{x x^{*}}(t, \tau)=\sum_{\alpha} R_{x x^{*}}^{\alpha}(\tau) \exp (j 2 \pi \alpha t)
$$

In (5), the real number $\alpha$ and the complex-valued functions $R_{x x^{*}}^{\alpha}(t, \tau)$ referred to as cycle frequency and cyclic autocorrelation, are frequencies and coefficients of the Fourier series expansion of $R_{x x^{*}}(t, \tau)$, for which cyclic autocorrelation function is defined as [5]

$$
R_{x x^{*}}^{\alpha}(\tau)=\lim _{T \rightarrow \infty} \frac{1}{T} \int_{-T / 2}^{T / 2} R_{x x^{*}}(t, \tau) \exp (-j 2 \pi \alpha t) d t
$$

The Fourier transform of $R_{x x^{*}}^{\alpha}(\tau)$ is called the cyclic spectrum (CS) which is defined as [5]

$$
S_{x x^{*}}^{\alpha}(f)=\int_{-\infty}^{\infty} R_{x x^{*}}^{\alpha}(\tau) \exp (-j 2 \pi \alpha \tau) d \tau
$$

Under the "narrowband" approximation, the time-varying component of the delay in the complex envelope $x(t-D(t))$ can be neglected [5]. In the proposed model to obtain cyclic autocorrelation and cyclic spectrum, the received signal is assumed as follows

$y_{r}=b_{d} x\left(t-D_{d}(t)\right)+b_{u} x\left(t-D_{u}(t)\right)$

Where in radar delay can be defined

$$
D(t)=2 R(t) / c=2\left(R_{0}+v t+a t^{2}\right) / c \longrightarrow D(t)=\kappa_{0}+\beta t+\gamma t^{2}
$$

In the delay expression, $R_{0}, v$ and $a$ are initial location, velocity and accelerate of target, respectively. In real time systems, the delay caused by the undesired is much less than the desired delay; in other words $D_{u}(t) \ll D_{d}(t)$, and can be taken constant. So $y_{r}(t)$ received 
signal is a mixture of the transmitted signal $x(t)$ and a chirp modulated version of $x(t)$ and can be written (8) as

$$
y_{r}=b_{u} x(t)+b_{d} x(t-\kappa) e^{j \pi\left(2 \beta t+\gamma t^{2}\right)}
$$

Where $b_{u}=a_{u} \exp \left(-j 2 \pi f_{c} R_{0 u}\right)$ is the complex gain from the undesired path, $b_{d}=a_{d} \exp \left(-j 2 \pi f_{c} R_{0 d}\right)$ is the complex gain from the target path, $\kappa=\kappa_{0}-D_{u}$ is interpreted as the difference between the constant part of $D_{d}(t)$, the constant $D_{u}(t), \beta=-2 \pi f_{c} v / c$ the frequency shift, and $\gamma=-4 f_{c} a / c$ the chirp rate. Thus, the autocorrelation function of $y(t)$ is given by

$$
\begin{aligned}
& R_{y y^{*}}(t, \tau)=E\left\{y_{r}^{*}(t) y_{r}(t+\tau)\right\} \\
& =E\left\{\left(b_{u}^{*} x^{*}(t)+b_{d}^{*} x^{*}(t-\kappa) e^{-j 2 \pi \beta t} e^{-j \pi \gamma t^{2}}\right)\left(b_{u} x(t+\tau)+b_{d} x(t+\tau-\kappa) e^{j 2 \pi \beta(t+\tau)} e^{j \pi \gamma(t+\tau)^{2}}\right)\right\} \\
& =E\left\{b_{u}^{2} x^{*}(t) x(t+\tau)+b_{d}^{*} b_{u} x(t+\tau) x^{*}(t-\kappa) e^{-j 2 \pi \beta t} e^{-j \pi \gamma t^{2}}+b_{u}^{*} b_{d} x^{*}(t) x(t+\tau-\kappa) e^{j 2 \pi \beta \tau} e^{j 2 \pi \beta t}\right. \\
& \left.\quad+b_{d}^{2} x(t+\tau-\kappa) x^{*}(t-\kappa) e^{j 2 \pi \beta \tau} e^{j \pi \gamma \tau^{2}} e^{j \pi \gamma \tau t}\right\}
\end{aligned}
$$

We assume $x(t)$ is almost-cyclostationary (ACS) [6], that is

$$
E\left\{x^{*}(t) x(t+\tau)\right\}=\sum_{\alpha \in \Gamma} R_{x x^{*}}^{(\alpha)}(\tau) \exp (-j 2 \pi \alpha t)
$$

where $\Gamma$ is the countable set of cycle frequencies and $\alpha_{n} \in \Gamma_{x x^{*}}$ Using (12) in (11) we have

$$
\begin{aligned}
& E\left\{y^{*}(t) y(t+\tau)\right\}=b_{u}^{2} \sum_{n} R_{x x^{*}}^{\alpha_{n}}(\tau) e^{-j 2 \pi \alpha_{n} t}+b_{d}^{2} \sum_{n} R_{x x^{*}}^{\alpha_{n}}(\tau) e^{j \pi\left(2 \beta \tau+\gamma \tau^{2}\right)} e^{j \pi\left(2 \alpha_{n}+\gamma \tau\right) t}\{\} \\
& +b_{u}^{*} b_{d} E\left\{x^{*}(t) x(t+\tau-\kappa) e^{j 2 \pi \beta \tau} e^{j 2 \pi \beta t}\right\}+b_{d}^{*} b_{u} E\left\{x^{*}(t-\kappa) x(t+\tau) e^{-j 2 \pi \beta t} e^{-j \pi \gamma t^{2}}\right\}
\end{aligned}
$$

If in the presented model, we assume $\kappa \simeq 0$ and $\eta_{n}=2 \alpha_{n}+\gamma \tau$ and address [7] as well, then equation (13) can be written as

$$
\begin{aligned}
& R_{y y^{*}}(\tau, \alpha)=b_{u}^{2} \sum_{n} R_{x x^{*}}^{\alpha_{n}}(\tau) e^{-j 2 \pi \alpha_{n} t}+b_{d}^{2} \sum_{n} R_{x x^{*}}^{\alpha_{n}}(\tau) e^{j \pi\left(2 \beta \tau+\gamma \tau^{2}\right)} e^{j \pi \eta_{n}(\tau) t} \\
& \quad+b_{u}^{*} b_{d} E\left\{x^{*}(t) x(t+\tau-\kappa) e^{j 2 \pi \beta \tau} e^{j 2 \pi \psi_{n} t}\right\}
\end{aligned}
$$

Where in (13), $\psi_{n}=\alpha_{n}+\beta$ and $\eta_{n}=2 \alpha_{n}+\gamma \tau$. The signal $y(t)$ has three cyclic frequencies, one of which depends on the lag or in other words, $\alpha_{y y^{*}}^{(1)}=-\beta, \alpha^{(2)} y^{*}{ }^{*}=-\gamma \tau / 2$ and $\alpha_{y y^{*}}^{(2)}=0$. 


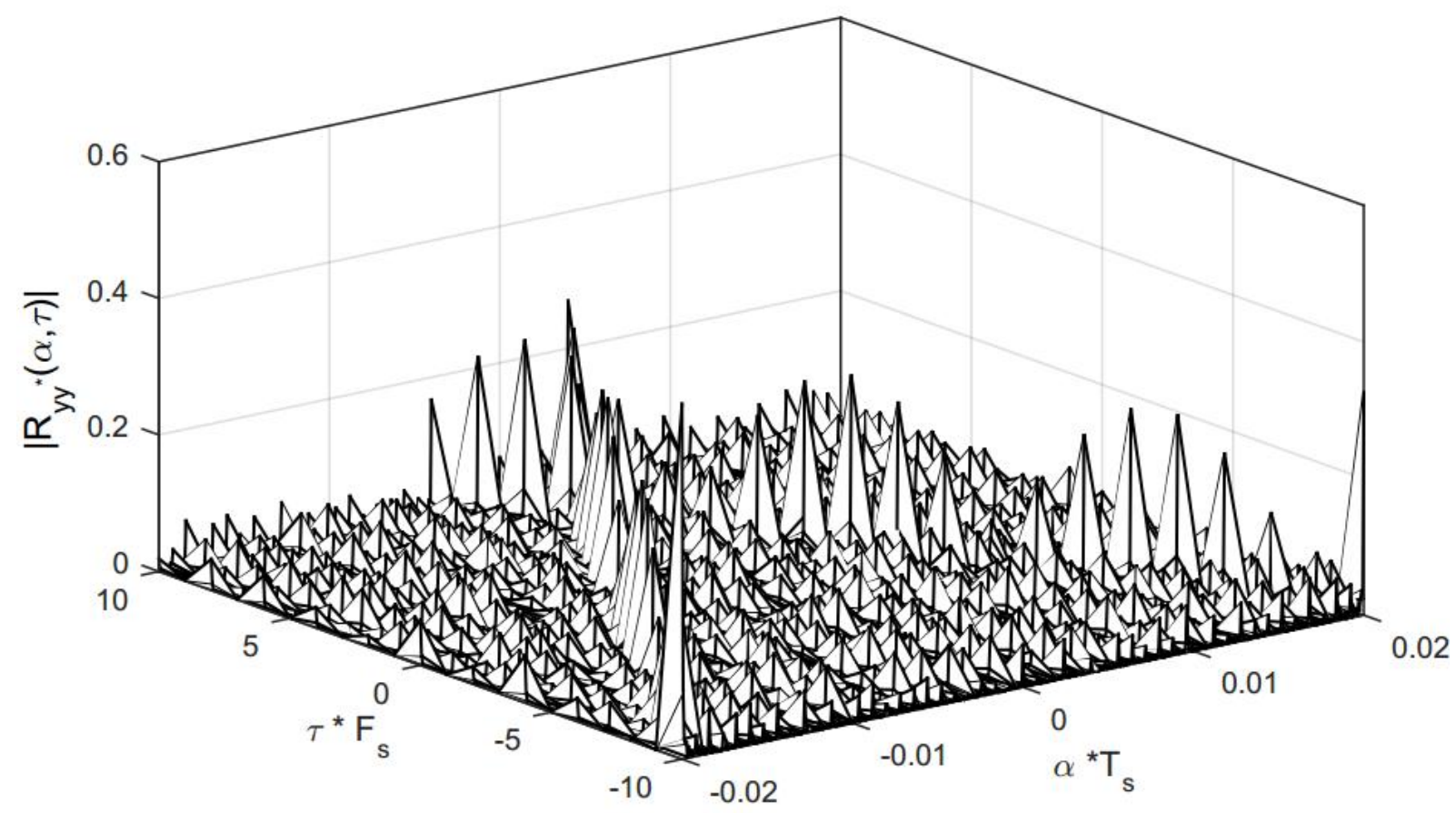

Fig.2. Cyclic correlogram mixture signal $y(t)$ in (10), undesired signal $S N R_{u}=10$ and desired signal $S N R_{d}=-5$

\section{SIMULATION AND RESULT}

In this section, the simulation experiments are explained through taking the theoretical results of the previous sections into consideration. The discrete-time sequences are obtained by uniformly sampling with period $T_{s}=1 / f_{s}$ as

$$
x(n) \equiv x(t)_{t=n T_{s}} \quad y(n) \equiv y(t)_{t=n T_{s}}
$$

Where, $x(t)$ and $y(t)$ are continuous Sinwave signals. The cyclic cross-correlation of the discrete-time sequence $y(n)$ and $x(n)$ at cycle frequency $\tilde{\alpha} \in\left[-\frac{1}{2}, \frac{1}{2}\right)$ are represented in [6].

The signal $y(t)$ passes through a Rayleigh Doppler Channel which produces a delay $\kappa_{0} \simeq 4000 T_{s}$, frequency shift $\beta=0.04 / T_{s}$. Cycle frequency is dependent on lag $\alpha_{x x^{*}}^{(2)}=-\gamma \tau / 2$ intercept in the point $(\alpha, \tau)=(0,0)$. By taking the mixture signal $y(t)$ in $(13)$ with the effect of $x(t)$ ACS into account, the effect of the chirp is rotated by angle $\theta$, where $\tan \theta=-\gamma / 2$ and a chirp rate $\gamma=-2 \times 10^{-3} / T_{s}^{2}$ that is shown in Fig.2. When $|t| \rightarrow \infty$, its cyclic autocorrelation functions are summable. So by increasing time, the best results will be obtained. If SNR of the undesired path increases, the peak of spectral increases as well, consequently if SNR desire path increases, the tail of spectral increases too and if the noise increases the whole surface increases. 


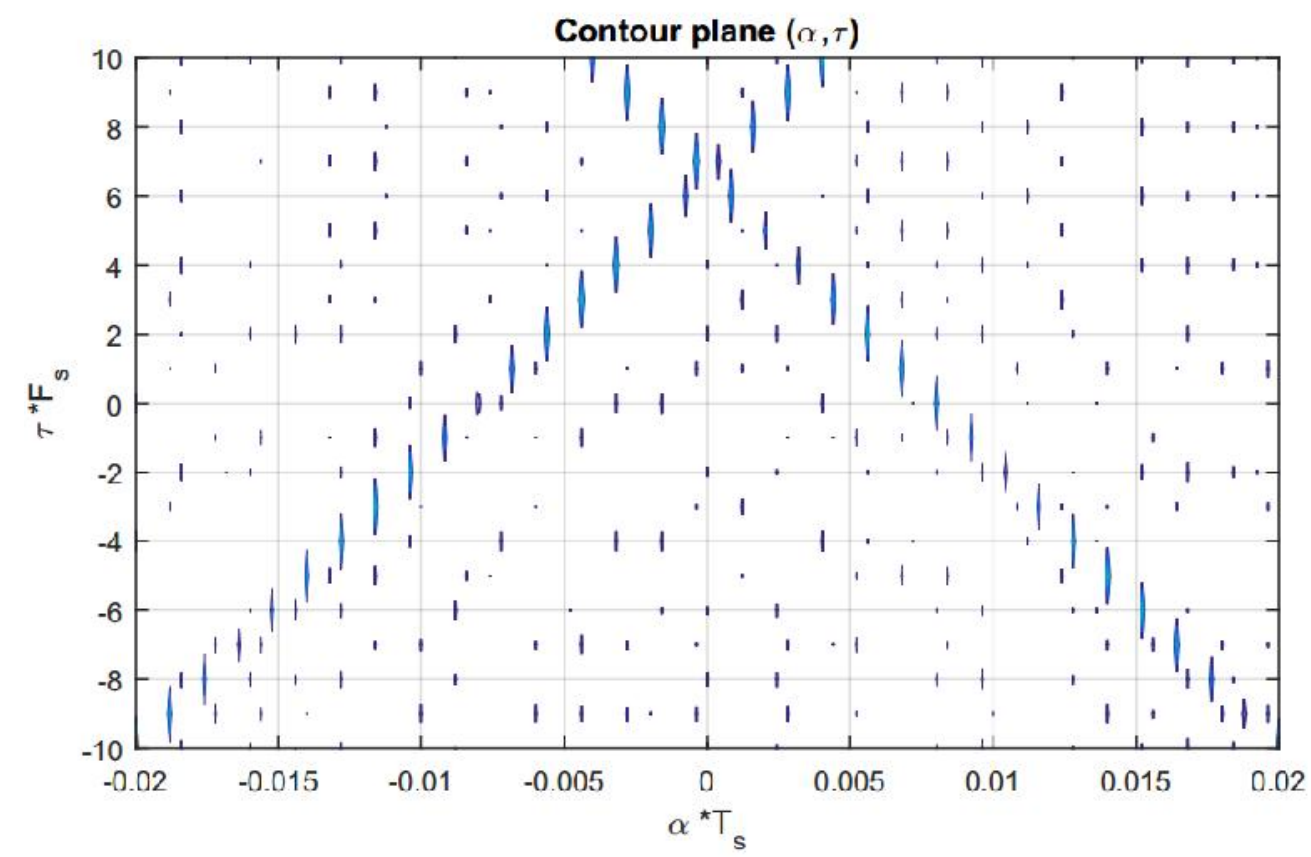

Fig.3. Contour plane $(\alpha, \tau)$ of cyclic spectrum, undesired signal $S N R_{u}=10 \mathrm{~dB}$ and desired signal $S N R_{d}=-5 \mathrm{~dB}$

Fig.2 shows the simulation of Monte Carlo. The obtained results from the simulation approve equation (13). In this simulation, the delay of $t_{d}=10^{-6}$ has been set and the total simulation time of $1000 t_{d}$ has been taken into account. As it is clear, the chirp rate of the initial frequency has been shown. Finally, if the noise level increases, the obtained slope becomes less clear and the signal level reduces to a level under the noise level.

\section{CONCLUSION}

This paper aimed to detect chirp rate and initial frequency of low signal in presence of the leakage signal using second-order statistical function of generalized almost-cyclostationary process. It was shown that cyclostationary methods compared to other methods, have less complexity in implementation and high precision. It was shown that the cyclostationary method in contrast with noise and interference has better performance in determining chirp signal feature.

\section{REFERENCES}

[1] Burt M. C., Burt M. P., Range Correlation Effects in Radars, Rec. of the IEEE National Radar Conference, April, 1993, 212-216.

[2] R. S. Ravent, Requirement on Master Oscillators for Coherent Radar, IEEE Proceedings, 
Vol. 54, No. 2,pp 237-243, February 1966.

[3] Beasley P. D. L., The Influence of Transmitter Phase Noise on FMCW Radar Performance, EuRAD, October, 2006, 331.

[4] Fu H., Kam P. Y. MAP/ML Estimation of the Frequency and Phase of a Single Sinusoid in Noise, IEEE Trans. Signal Process., Vol. 55, No. 3, pp.834-845, 2007.

[5] lzzo L., Napolitano A. Higher-order Theory of Generalized almost-Cyclostationary times-series, IEEE Trans. Signal Process., Vol. 46, No. 11, pp.2975-2989, 1998.

[6] Gardner W. A., Statistical Spectral Analysis: A Nonprobabilistic Approach, PrenticeHall, 1988.

[7] Napolitano A. Generalizations of Cyclostationary Signal Processing: Spectral Analysis and Application, Wiley-IEEE Press, December, 2012.

[8] Napolitano A. Estimation of Second-Order Cross-Moments of Generalized AlmostCyclostaionary Process, IEEE Trans. Inf. Theory, Vol. 53, No. 6, pp.2204-2228, 2007.

[9] Napolitano A., Cyclostationary: Limits and Generalizations, Signal Processing, in Press, 2016.

[10] Giannakis G. B., Madisetti V. K. and Williams D. B. Cyclostationary Signal Analysis, in The Handbook of Digital Signal Processing, CRC Press, Inc. and IEEE Press, 1998.

[11] Giannakis G. B, Madisetti V. K and Williams D. B. Cyclostationary Signal Analysis”, in The Handbook of Digital Signal Processing, CRC Press, Inc. and IEEE Press, 1998.

[12] Gardner W. A., Napolitano A., and Paura L. Cyclostationarity: Half a Century of Research. Signal Processing, Vol. 86, pp. 639-697, April 2006.

[13] Napolitano A. Generalizations of Cyclostationary Signal Processing: Spectral Analysis and Applications. John Wiley \& Sons, Ltd. - IEEE Press, 2012.

[14]Gardner W. A. Introduction to Random Processes with Applications to Signals and Systems.New York: McGraw-Hill, $2^{\text {nd }}$., 1990.

[15]Dehay D., Le'Skow J., Napolitano A. Central Limit Theorem in the Functional Approach, IEEE Trans.on Signal Process., Vol. 61, pp. 4025-4037, June, 2013.

[16]Huang Z. T, Zhou Y. Y., Jiang W. L., Lu Q. Z. Joint Estimation of Doppler and TimeDifference-of-Arrival Exploiting Cyclostationary Property, IEE Proceedings - Radar, Sonar and Navigation, Vol.149, pp.161-165, 2002.

[17] Gardner W. A., Cyclostationarity in Communications and Signal Processing, IEEE Press, Piscataway, NJ, 1994.

[18] Sutton P. D, Nolan K.E and Doyle L. E. Cyclostationary Signatures in Practical 
Cognitive Radio Applications, IEEE Journal on Selected Areas in Communications, Vol. 26, No. 1, pp. 13-24, 2008.

[19] Spooner C. M and Gardner W. A. The Cumulant Theory of Cyclostationary Time-Series, Part II: Development and Applications, IEEE Transactions on Signal Processing. Vol. 42, No. 12, pp. 3409-3429, Dec. 1994.

[20] Gardner W. A. Exploitation of Spectral Redundancy in Cyclostationary Signals, IEEE Signal Processing Magazine, Vol. 8, No. 2, pp. 14-36, April 1991.

[21] Gardner W. A., Spooner C. M. The Cumulant Theory of Cyclostationary Time-Series, Part I: Foundation, IEEE Trans. Signal Processing, Vol. 42, pp. 3387-3408, Dec. 1994.

[22] Izzo L and Napolitano A. Effects of Random Linear Transformations on Higher-Order Cyclostationary Time-Series, in Proc. Twenty-Ninth Annu. Asilomar Conf. Signals, Syst., Comput. , Pacific Grove, CA, Oct. 1995.

\section{How to cite this article:}

Heydari K, Azmi P, Abbasi B and Heydari A. Determining the parameters of chirp signals using cyclostationary method in presence of the interference. J. Fundam. Appl. Sci., 2016, $8(2 S), 478-486$. 